\title{
Room temperature, continuous wave lasing in microcylinder and microring quantum dot laser diodes
}

Cite as: Appl. Phys. Lett. 100, 031111 (2012); https://doi.org/10.1063/1.3678031

Submitted: 09 November 2011 • Accepted: 28 December 2011 • Published Online: 20 January 2012

M. Munsch, J. Claudon, N. S. Malik, et al.

ARTICLES YOU MAY BE INTERESTED IN

Whispering gallery mode lasing in high quality GaAs/AIAs pillar microcavities Applied Physics Letters 96, 071103 (2010); https://doi.org/10.1063/1.3315869

Whispering gallery mode lasing in electrically driven quantum dot micropillars Applied Physics Letters 97, 101108 (2010); https://doi.org/10.1063/1.3488807

Whispering-gallery mode microdisk lasers

Applied Physics Letters 60, 289 (1992); https://doi.org/10.1063/1.106688

田QBLOX

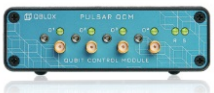

1 qubit
Shorten Setup Time Auto-Calibration More Qubits

\section{Fully-integrated} Quantum Control Stacks Ultrastable DC to $18.5 \mathrm{GHz}$ Synchronized $<<1$ ns Ultralow noise

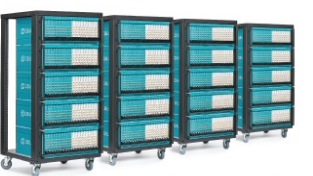

100s qubits

visit our website > 


\title{
Room temperature, continuous wave lasing in microcylinder and microring quantum dot laser diodes
}

\author{
M. Munsch, ${ }^{1}$ J. Claudon, ${ }^{1, a)}$ N. S. Malik, ${ }^{1}$ K. Gilbert, ${ }^{2}$ P. Grosse, ${ }^{2}$ J.-M. Gérard, ${ }^{1}$ F. Albert, ${ }^{3}$ \\ F. Langer, ${ }^{3}$ T. Schlereth, ${ }^{3}$ M. M. Pieczarka, ${ }^{3}$ S. Höfling, ${ }^{3}$ M. Kamp, ${ }^{3}$ A. Forchel, ${ }^{3}$ \\ and S. Reitzenstein ${ }^{3,4}$ \\ ${ }^{1}$ CEA-CNRS-UJF group "Nanophysique et Semiconducteurs," CEA, INAC, SP2M, F-38054 Grenoble, France \\ ${ }^{2}$ CEA, LETI, MINATEC Campus, 17 rue des Martyrs, 38054 Grenoble, France \\ ${ }^{3}$ Technische Physik, Physikalisches Institut, Universität Würzburg, Am Hubland, D-97074 Würzburg, Germany \\ ${ }^{4}$ Institut für Festkörperphysik, Technische Universität Berlin, Hardenbergstraße 36, D-10623 Berlin, Germany
}

(Received 9 November 2011; accepted 28 December 2011; published online 20 January 2012)

We demonstrate room temperature, continuous wave lasing of laser diodes based on AlGaAs whispering gallery mode (WGM) resonators (microcylinder and microring) embedding a quantum dot (QD) active layer. Using InGaAlAs QDs, high-Q (>60 000) lasing modes are observed around $910 \mathrm{~nm}$, up to $50^{\circ} \mathrm{C}$. Lasing with similar performance is obtained around $1230 \mathrm{~nm}$, using InAs QDs. Furthermore, we show that the current injection in the active part of the device is improved in ring resonators, leading to threshold currents of approximately $4 \mathrm{~mA}$ for a device with $80 \mu \mathrm{m}$ diameter. This geometry also suppresses WGMs with a high radial order, thus simplifying the lasing spectra. In these conditions, stable single-mode and two-color lasing can be obtained. (C) 2012 American Institute of Physics. [doi:10.1063/1.3678031]

Over the last two decades, whispering gallery mode (WGM) optical resonators made of semiconductor material have attracted a considerable interest. Such microcavities confine optical fields over tiny volumes (a few cubic wavelengths), while displaying a photon storage time approaching $10^{5}$ optical cycles. ${ }^{1}$ They have led to the demonstration of fundamental quantum electrodynamics effects at cryogenic temperature, using a quantum dot (QD) as an integrated quantum light source. ${ }^{2-4}$ On the application side, these resonators are promising candidates for the development of microlasers. They can be integrated in planar photonic circuits, ${ }^{5}$ and various strategies have been explored to obtain a directive far-field emission. ${ }^{6}$ For practical applications, electrical pumping and room temperature (RT) operation are highly desirable. To date, most WGM laser diodes have exploited a quantum well gain medium, in III-V materials with low surface losses. ${ }^{7,8}$ Nevertheless, self-assembled QDs offer an alternative with several advantages. In particular, the gain curve which is inhomogeneously broadened by the QD size dispersion allows for two-color lasing. This is a key ingredient of a recently proposed integrated $\mathrm{THz}$ source ${ }^{9}$ based on intra-cavity difference frequency generation.

Until recently, most QD WGM microlasers were based on a suspended $\mu$-disk supported by a low diameter pedestal. ${ }^{1,10-14}$ This geometry maximises the confinement of the optical field but suffers from poor heat sinking, a severe drawback to achieve continuous wave (CW) lasing. ${ }^{2,10}$ Moreover, it does not allow for a direct injection of charge carriers at the location of the WGM. Consequently, RT CW lasing in a QD- $\mu$-disk system has only been achieved under optical pumping. ${ }^{1,13}$ In this context, AlGaAs $\mu$-cylinders have recently emerged as a new class of high performance optical WGM resonators. ${ }^{15,16}$ Compared to suspended $\mu$-disks, $\mu$-cylinders provide efficient heat sinking and

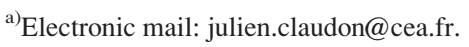

straightforward carrier injection. Taking advantage of these assets, cryogenic $\mathrm{CW}$ microlasers were rapidly demonstrated, using optical ${ }^{17,18}$ or electrical ${ }^{19}$ carrier injection.

In this letter, we demonstrate RT CW laser diodes based on high-Q AlGaAs $\mu$-cylinder and $\mu$-ring resonators. We show results obtained from two families of devices, embedding InGaAlAs QDs and InAs quantum dot-in-a-well (DWELL) that were optimized for operation at elevated temperatures. The first device family exhibits WGM lasing around $910 \mathrm{~nm}$ with Q-factors exceeding 60000 . In the second device family, lasing occurs around $1230 \mathrm{~nm}$, with similar performance. We furthermore show that ring resonators exhibit smaller lasing thresholds $(\sim 4 \mathrm{~mA})$ and a simpler lasing spectrum. Under these conditions, stable single-mode and two-color lasing can be obtained.

Our devices are made out of planar structures which are grown by molecular beam epitaxy on a (001) n-doped GaAs wafer. The active layer of sample 1 (S1) consists in a high gain, single layer of self-assembled InGaAlAs QDs emitting at about $900 \mathrm{~nm} .^{20}$ This layer is inserted in a $260 \mathrm{~nm}$-thick $\mathrm{AlGaAs}$ layer, whose $\mathrm{Al}$ content increases from $18 \%$ to $30 \%$ as the distance to the active layer increases. To ensure vertical photonic confinement, this central layer is sandwiched by $\mathrm{Al}_{0.3} \mathrm{Ga}_{0.7}$ As claddings. Sample 2 (S2) employs a 6 layer InGaAs DWELL design ${ }^{21}$ with an emission peak at about $1270 \mathrm{~nm}$. The active layer is inserted in a $250 \mathrm{~nm}$-thick GaAs waveguide which is embedded in $\mathrm{Al}_{0.4} \mathrm{Ga}_{0.6} \mathrm{As}$ claddings. The RT modal gain of S1 $\left(63 \mathrm{~cm}^{-1}\right)$ and S2 $\left(17 \mathrm{~cm}^{-1}\right)$ were estimated by defining a series of ridge waveguide lasers with various resonator lengths. The bottom and top AlGaAs layers are, respectively, $n$ - and p-doped with reduced doping concentration close to the central part of the waveguide. Lateral photonic confinement is achieved by etching $\mu$-cylinders or $\mu$-rings in the samples. The etching mask (Ti-Pt-Au-Ni) is first deposited on the epitaxial structure using electron beam lithography, metal evaporation, and acetone lift-off. After 


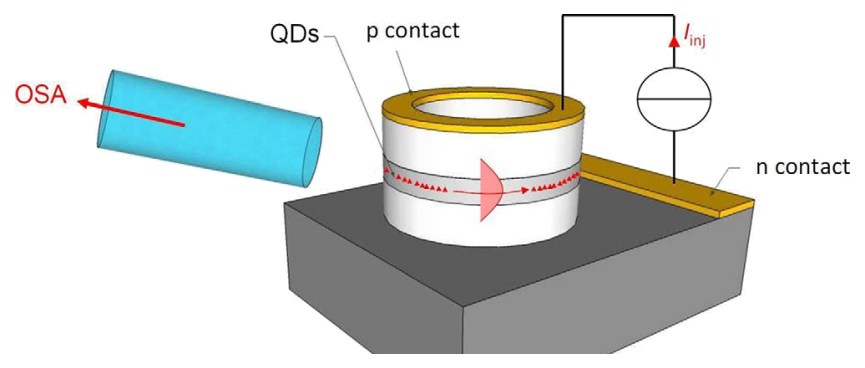

FIG. 1. (Color online) Sketch of a $\mu$-ring laser diode and experimental configuration.

partial removal of the top Ni layer during the reactive ion etching (RIE) step, it further acts as top electrode. The ncontact (Ni-Ge-Au-Ni-Au) is deposited onto the substrate, prior to a 2 min annealing at $400^{\circ} \mathrm{C}$.

Optical and electrical characterizations are performed at RT; the experimental configuration is sketched in Fig. 1. Electrical contacts are established using two sharp metallic tips (curvature radius of $2 \mu \mathrm{m}$ ). The devices are connected to a CW current source, also used to measure the intensityvoltage characteristics. The emission of the devices is partially collected by the cleaved facet of a multimode optical fiber (core diameter: $50 \mu \mathrm{m}$, numerical aperture $=0.2$ ). To optimize the collection of light, the fiber is approached close to the resonator $(\sim 20 \mu \mathrm{m})$, making a small angle $\left(\sim 10^{\circ}\right)$ with the equatorial plane of the sample. The collected light is then analysed with an optical spectrum analyser (OSA), with a maximum resolution of $10 \mathrm{pm}$.

Figures 2(a) and 2(c) show the spectra of two laser diodes based on $\mu$-cylinder resonators, for several injection currents $I_{\mathrm{inj}}$. They are made out of S1 and S2 and feature a
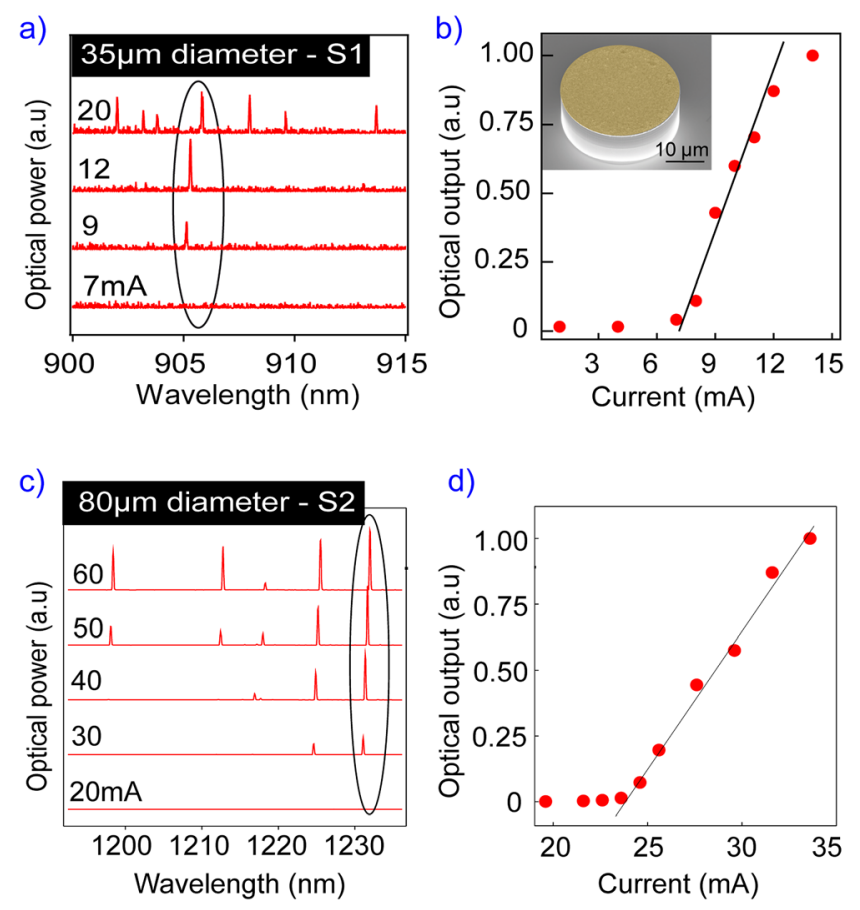

FIG. 2. (Color online) Room temperature, CW lasing of two $\mu$-cylinder laser diodes. They are fabricated from S1 and S2 and feature a diameter of $35 \mu \mathrm{m}$ and $80 \mu \mathrm{m}$, respectively. (a) and (c) Waterfall plot of the spectra of the laser diodes for increasing injection current $I_{\text {inj. }}$ (b) and (d) Output power of the brightest lasing mode vs $I_{\text {inj. }}$. A SEM picture of a representative $35 \mu \mathrm{m}$ diameter device is shown in the inset. diameter $D=35 \mu \mathrm{m}$ and $80 \mu \mathrm{m}$, respectively. In both cases, a clear evidence of multimode lasing under $\mathrm{CW}$ pumping at room temperature is found. Lasing modes are observed around $\lambda=905 \mathrm{~nm}$ for $\mathrm{S} 1$ and around $\lambda=1230 \mathrm{~nm}$ for S2. For both devices, the number of simultaneously lasing modes increases with $I_{\text {inj. }}$. The integrated intensity of the brightest mode of S1 is plotted in Fig. 2(b), and we determine a threshold current of $7.5 \mathrm{~mA}$. For S2, the threshold current is larger $(24 \mathrm{~mA})$, owing to the larger surface of active material [Fig. 2(d)]. The total optical output power of this multimode device is about $1 \mathrm{~mW}$ at $I_{\mathrm{inj}}=100 \mathrm{~mA}$ (not shown).

The dipole associated with the fundamental optical transition of the QDs is perpendicular to the growth axis, which coincides with the resonator axis. The QD gain medium, thus, feeds optical modes with a TE polarization, which are well defined in a $\mu$-cylinder resonator. ${ }^{16} \mathrm{~A}$ WGM resonance is then identified by a set of three integers $(z, r, m)$ which are, respectively, the vertical, radial, and azimuthal indices. ${ }^{22} \mathrm{We}$ calculate the resonance wavelength of these modes in the frame of the so-called effective index approximation, which decouples the description of the vertical and lateral photonic confinements. Here, only the $z=1$ modes are confined. For the device defined in $\mathrm{S} 1$ (S2), the $\mathrm{TE}_{1,1, m}$ modes coupled to the QD luminescence exhibit $m$ around 390 (670). A complete and unambiguous identification of $r$ and $m$ in the $\mu$-cylinders resonance spectra is difficult, because of the relatively large spectral density of $\mathrm{TE}_{1, r, m}$ modes. In addition, using the simple model of Ref. 17, we estimate a spontaneous emission coupling $\beta$-factor of a few $10^{-4}$ for the QDs coupled to the lasing modes. This order of magnitude is consistent with the resonator modal volume and the homogeneous linewidth of the emitters $\left(\sim 5 \mathrm{~nm}\right.$ at room temperature $\left.{ }^{23}\right)$.

WGMs with a low radial index $(r=1)$ are located at the periphery of the structure, with a typical lateral extension of one wavelength. Thus, the central part of the gain medium does not participate to WGM lasing and current flowing to this area is lost, only generating additional heat. We thus fabricated microlasers with a ring shaped cross-section out of S1 and S2. In S2, the $\mu$-rings were defined directly by the RIE step. In S1, the $\mu$-rings are defined with a focus ion beam (FIB) milling, starting from plasma-etched $\mu$-cylinders. The ring is isolated from the central part by a trench which ends at the QD active layer. In addition, the top of the central part has been etched over half a micron. This geometry allows contacting tiny rings (wall thicknesses as low as $3 \mu \mathrm{m})$ with the tip, while avoiding spurious contact to the central part. In addition, this structuration combines selective injection of the holes at the periphery of the resonator with a minimal degradation of its electrical and thermal resistances.

Figure 3(a) shows the threshold currents of a series of $\mu$-ring laser diodes made out of S2. They feature an outer diameter of $80 \mu \mathrm{m}$ and decreasing wall thicknesses $(20,15$, and $10 \mu \mathrm{m})$. We observe a significant drop of the lasing threshold down to $5.3 \mathrm{~mA}$ as the central part of the $\mu$-cylinder is removed. The threshold dependence versus the temperature of another microlaser with a wall thickness of $10 \mu \mathrm{m}$ is illustrated in Fig. 3(b). CW lasing is observed up to $50^{\circ} \mathrm{C}$, and a threshold current as low as $4.2 \mathrm{~mA}$ for $10^{\circ} \mathrm{C}$ was achieved. 

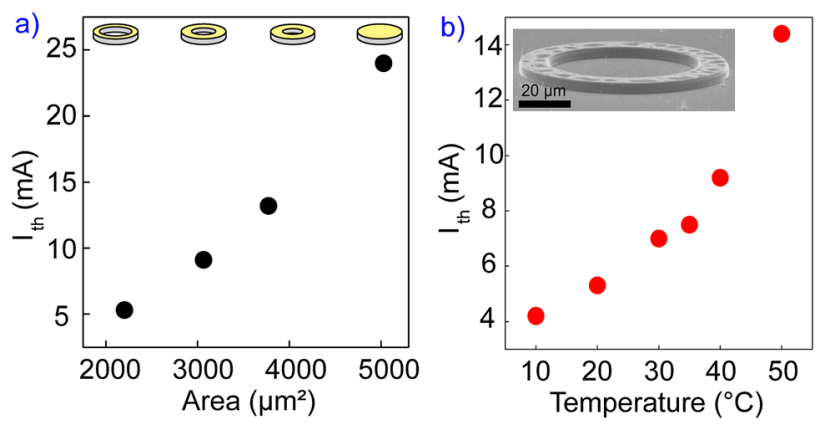

FIG. 3. (Color online) (a) Threshold current versus surface for different resonators with an outer diameter of $80 \mu \mathrm{m}$ ( $\mu$-disk and $\mu$-rings with a wall thickness of 20,15 , and $10 \mu \mathrm{m}$ ). (b) Temperature dependence of the threshold current of a $\mu$-ring laser with a wall thickness of $10 \mu \mathrm{m}$ (SEM image in the inset).

In addition, the patterning into a $\mu$-ring helps suppressing the modes with a high radial order. The lasing spectrum is then dominated by the $\mathrm{TE}_{1,1, m}$ modes, for which $m$ is determined with an uncertainty of 1 . Figure 4(a) shows the lasing spectra of a ring resonator defined in S1, for increasing injection current. The device features an outer diameter $D=35 \mu \mathrm{m}$ and a wall thickness of $3 \mu \mathrm{m}$ [inset in Fig. 4(c)]. A clean single-mode lasing of the $\mathrm{TE}_{1,1,390}$ mode is observed for $I_{\text {inj }}$ between $4 \mathrm{~mA}$ and $7 \mathrm{~mA}$. Note the lasing threshold in the $\mu$-ring is nearly a factor 2 smaller than the original $\mu$-cylinder laser. Figure 4(b) illustrates the high spectral purity of the laser emission, with Q-factors exceeding 60000. The mode is split, a commonly observed feature in high-Q WGM resonators. ${ }^{1,17,18,24}$ Indeed, a very small perturbation
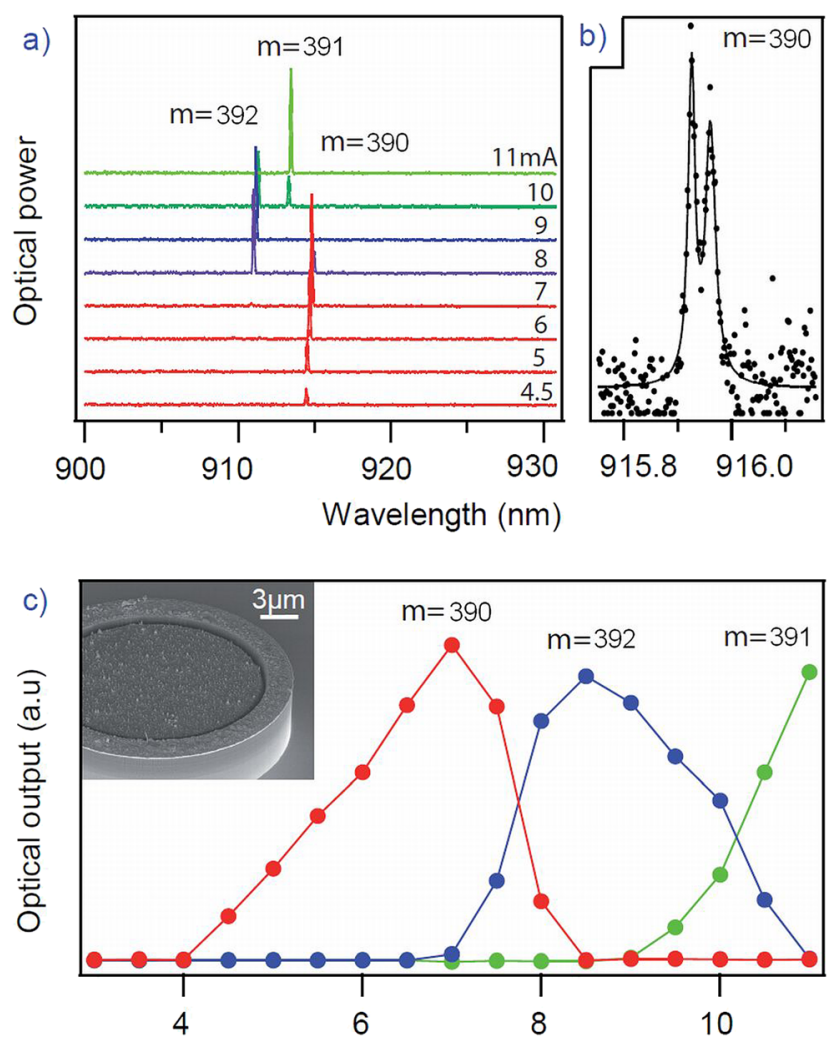

FIG. 4. (Color online) Room temperature, $\mathrm{CW}$ lasing of a $\mu$-ring laser diode made out of S1 (outer diameter: $35 \mu \mathrm{m}$, wall thickness: $3 \mu \mathrm{m}$ ). (a) Waterfall plot of the spectra for increasing injection current $I_{\text {inj. }}$ (b) High-resolution spectrum for $I_{\text {inj }}=5 \mathrm{~mA}$. (c) Output power of the first three lasing modes vs

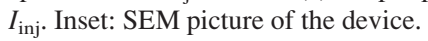

introduced by a residual sidewall roughness ${ }^{24}$ or due to the refractive index inhomogeneity associated with the QDs (Refs. 18 and 25) is sufficient to couple counter-propagating $\pm m$ modes and to generate a sizeable splitting.

As shown in Figs. 4(a) and 4(c), the simultaneous lasing of the $\mathrm{TE}_{1,1,392}$ and $\mathrm{TE}_{1,1,390}$ modes is obtained for $I_{\text {inj }}$ between 7.0 and $8.5 \mathrm{~mA}$. Similar lasing intensities in the two modes are obtained for $I_{\mathrm{inj}}=7.7 \mathrm{~mA}$. Such a two-color lasing is particularly relevant for a recently proposed scheme for $\mathrm{THz}$ generation, ${ }^{9}$ where $\mathrm{THz}$ is generated by non-linear frequency difference between two lasing WGMs in the near infrared. Above $10 \mathrm{~mA}$, the $\mathrm{TE}_{1,1,391}$ appears and dominates the spectrum for $I_{\mathrm{inj}}>11 \mathrm{~mA}$. The free spectral range $\Delta \lambda$ between the modes $\mathrm{TE}_{1,1,390}, \mathrm{TE}_{1,1,391}$, and $\mathrm{TE}_{1,1,392}$ is measured at $1.98 \mathrm{~nm}$. This value is in very good agreement with the predicted value of $1.97 \mathrm{~nm}$, obtained with the expression $\Delta \lambda=\lambda^{2} /\left(\pi D n_{g}\right)$, where $n_{g}=3.820$ is the group index of the $\mathrm{TE}_{z=1}$ mode guided in the planar (unetched) structure.

The ring patterning increases the series resistance from $8 \Omega$ to $38 \Omega$, but this does not compromise the lasing performance. In fact, the ring geometry still provides a very efficient heat management. From lasing measurements conducted between $20^{\circ} \mathrm{C}$ and $50^{\circ} \mathrm{C}$ (not shown), we estimate a temperature shift for the mode wavelength of $+65 \mathrm{pm} /{ }^{\circ} \mathrm{C}$. When $I_{\mathrm{inj}}$ is increased from $4 \mathrm{~mA}$ to $8 \mathrm{~mA}$, we measure a redshift as low as $480 \mathrm{pm}$, corresponding to a temperature increase of $7^{\circ} \mathrm{C}$.

In conclusion, we have demonstrated RT WGM laser diodes using $\mu$-cylinder and $\mu$-ring resonators, with a QD gain medium at approximately $900 \mathrm{~nm}$ and $1230 \mathrm{~nm}$. Ring resonators provide the best performance in terms of lasing threshold and lasing spectrum. Thanks to the inhomogeneously broadened gain provided by the QDs, the lasing can be turned from single-mode to two-color, simply by changing the injection current.

The authors warmly thank J. F. Motte, M. Terrier, and M. Emmerling for expert technical assistance, and A. Tchelnokov for his support. The authors acknowledge the financial support of the Future and Emerging Technologies (FET) programme within the 7th Framework Programme for Research of the European Commission, under the FET-Open "TREASURE" project (Grant No. 250056).

${ }^{1}$ K. Srinivasan, M. Borselli, T. J. Johnson, P. E. Barclay, O. Painter, A. Stintz, and S. Krishna, Appl. Phys. Lett. 86, 151106 (2005).

${ }^{2}$ B. Gayral, J. M. Gérard, A. Lemaître, C. Dupuis, L. Manin, and J. L. Pelouard, Appl. Phys. Lett. 75, 1908 (1999).

${ }^{3}$ A. Kiraz, P. Michler, C. Becher, B. Gayral, A. Imamoglu, L. Zhang, E. Hu, W. V. Schoenfeld, and P. M. Petroff, Appl. Phys. Lett. 78, 3932 (2001).

${ }^{4}$ E. Peter, P. Senellart, D. Martrou, A. Lemaître, J. Hours, J. M. Gérard, and J. Bloch, Phys. Rev. Lett. 95, 067401 (2005).

${ }^{5}$ D. Liang and J. E. Bowers, Nature Photon. 4, 511 (2010).

${ }^{6}$ L. Mahler, A. Tredicucci, F. Beltram, C. Walther, J. Faist, B. Witzigmann,

H. E. Beere, and D. A. Ritchie, Nature Photon. 3, 46 (2008).

${ }^{7}$ A. F. J. Levi, R. E. Slusher, S. L. McCall, T. Tanbun-Ek, D. L. Coblentz, and S. J. Pearton, Electron. Lett. 28, 1010 (1992).

${ }^{8}$ M. Fujita, A. Sakai, and T. Baba, IEEE J. Sel. Top. Quantum Electron. 5, 673 (1999).

${ }^{9}$ A. Andronico, J. Claudon, J.-M. Gérard, V. Berger, and G. Leo, Opt. Lett. 33, 2416 (2008).

${ }^{10}$ S. M. K. Thiyagarajan, A. F. J. Levi, C. K. Lin, I. Kim, P. D. Dapkus, and S. J. Pearton, Electron. Lett. 24, 2333 (1998).

${ }^{11}$ H. Cao, J. Y. Xu, W. H. Xiang, Y. Ma, S.-H. Chang, S. T. Ho, and G. S. Solomon, Appl. Phys. Lett. 76, 3519 (2000). 
${ }^{12}$ P. Michler, A. Kiraz, L. Zhang, C. Becher, E. Hu, and A. Imamoglu, Appl. Phys. Lett. 77, 184 (2000).

${ }^{13}$ T. Ide and T. Baba, Opt. Express 13, 1615 (2005).

${ }^{14}$ M.-H. Mao, H.-C. Chien, J.-Z. Hong, and C.-Y. Cheng, Opt. Express 19, 14145 (2011).

${ }^{15}$ V. N. Astratov, S. Yang, S. Lam, B. D. Jones, D. Sanvitto, D. M. Whittaker, A. M. Fox, M. S. Skolnick, A. Tahraoui, P. W. Fry, and M. Hopkinson, Appl. Phys. Lett. 91, 071115 (2007).

${ }^{16}$ Y.-R. Nowicki-Bringuier, J. Claudon, C. Böckler, S. Reitzenstein, M. Kamp, A. Morand, A. Forchel, and J. Gérard, Opt. Express 15, 17291 (2007).

${ }^{17}$ P. Jaffrennou, J. Claudon, M. Bazin, N. S. Malik, S. Reitzenstein, L. Worschech, M. Kamp, A. Forchel, and J.-M. Gérard, Appl. Phys. Lett. 96, 071103 (2010).
${ }^{18}$ B. D. Jones, M. Oxborrow, V. N. Astratov, M. Hopkinson, A. Tahraoui, M. S. Skolnick, and A. M. Fox, Opt. Express 18, 22578 (2010).

${ }^{19}$ F. Albert, T. Braun, T. Heindel, C. Schneider, S. Reitzenstein, S. Höfling, L. Worschech, and A. Forchel, Appl. Phys. Lett. 97, 101108 (2010).

${ }^{20}$ T. W. Schlereth, C. Schneider, W. Kaiser, S. Höfling, and A. Forchel, Appl. Phys. Lett. 90, 221113 (2007).

${ }^{21}$ R. Krebs, S. Deubert, J. Reithmaier, and A. Forchel, J. Cryst. Growth 251, 742 (2003).

${ }^{22}$ N. C. Frateschi and A. F. J. Levi, J. Appl. Phys. 80, 644 (1996).

${ }^{23}$ P. Borri, W. Langbein, S. Schneider, U. Woggon, R. L. Sellin, D. Ouyang, and D. Bimberg, Phys. Rev. Lett. 87, 157401 (2001).

${ }^{24}$ M. Borselli, K. Srinivasan, P. E. Barclay, and O. Painter, Appl. Phys. Lett. $\mathbf{8 5}, 3693$ (2004).

${ }^{25}$ K. R. Hiremath and V. N. Astratov, Opt. Express 16, 5421 (2008). 